\title{
Study on the Detectability of the Sky-Surface Wave Hybrid Radar
}

\author{
Hou Chengyu, ${ }^{1}$ Ke Guo, ${ }^{1}$ Shi Tiange, ${ }^{2}$ and Wang Yuxin ${ }^{3}$ \\ ${ }^{1}$ School of Electronics and Information Engineering, Harbin Institute of Technology, Harbin, Hei Longjiang 150001, China \\ ${ }^{2}$ School of Astronautics, Harbin Institute of Technology, Harbin, Hei Longjiang 150001, China \\ ${ }^{3}$ Institute of Electronic and Information Engineering, Harbin Institute of Technology, Harbin, Hei Longjiang 150001, China
}

Correspondence should be addressed to Hou Chengyu; houcy@hit.edu.cn

Received 23 April 2014; Accepted 10 June 2014; Published 6 July 2014

Academic Editor: Hamid R. Karimi

Copyright (C) 2014 Hou Chengyu et al. This is an open access article distributed under the Creative Commons Attribution License, which permits unrestricted use, distribution, and reproduction in any medium, provided the original work is properly cited.

\begin{abstract}
Working in the HF (high-frequency) band and the transmitter and receiver locating separately, the sky-surface wave hybrid radar both has the capabilities of the OTHR (over-the-horizon radar) and the advantage of the bistatic radar. As the electromagnetic wave will be disturbed by the ionosphere, interfered by the sea clutter and attenuated by the sea surface, the detectability of this radar system is more complex. So, in this paper, we will discuss the problem detailedly. First of all, the radar equation is deduced based on the propagation of the electromagnetic wave. Then, how to calculate the effect of the ionosphere and the propagation loss is discussed. And an example based on the radar equation is given. At last, the ambiguity function is used to analyze the range and velocity resolution. From the result, we find that the resolution has relation with the location of the target and the height of reflection point of the ionosphere. But compared with the location, the effect of the ionospheric height can be ignored.
\end{abstract}

\section{Introduction}

Sky-wave and surface-wave OTH radar take advantage of characteristics of HF electromagnetic wave refraction by the ionosphere and diffraction on the sea surface respectively to achieve the targets detection. The sky-surface wave hybrid radar is a new type of radar systems, which has strong application prospects on detecting the stealth target, countering antiradiation missiles, enhancing the radar survivability, and other aspects. However, the detectability of single sky-wave or ground-wave radar is difficult to improve. So applying the MIMO (multi input multi output) radar theory into the sky-wave and ground-wave radar system to improve the performance of the OTH radar is one of new research fields in recent years [1].

The sky-surface wave hybrid radar is a MIMO radar system. This system is based on the technology of the existing sky-wave and ground-wave OTH radar system and works as a bistatic radar. So the detectability and target recognition performance can be further improved. In this system, the transmitter of the sky-wave radar is located inland far away from the coast, while the receiver of the ground-wave radar is on the coast, as the coastal part of the system only receives the echo, which prevents the receiver from the antiradiation missile. Meanwhile, the multireceivers receive the target echoes from different directions, which can effectively solve the problem of RCS (Radar Cross Section) flickering and improve detectability of the low-altitude target.

Nowadays, this hybrid radar is at the experimental stage. Papers [2-4] describe the research status of this system in Canada, USA, and Australia. From these papers, we find that the test systems are built with the same working principle. These systems transmit the wave with the sky wave mode and the receivers are placed close to the target. Since the receivers are close to the target, the target echo is relatively stronger which can improve the detection probability. Researchers in Wuhan University try to use the broadcast signal DRM to realize the target detection in the hybrid mode [5]. As a new radar system, only the sea clutter characteristics, the ionospheric influence on the resolution, and the time 
availability and the asynchronous filtering are mentioned in papers [6-10]. Few works on the analysis of the radar detectability have been reported. So we will discuss this topic thoroughly in this paper.

This paper mainly studies two aspects of the radar detectability. On one hand, the hybrid sky-surface wave radar equation is deduced. Then the attenuation effect of the ionosphere and sea surface on the radar detectability is analyzed. And an example is given to illustrate how to use the radar equation to calculate the detection range. On the other hand, the ambiguous function of the hybrid radar with FMCW (frequency modulated continuous wave) is analyzed and used to study the range and velocity resolutions of the hybrid sky-surface wave radar. And the results are compared with the ones of monostatic OTH radar.

\section{The Radar Equation and the Propagation Loss}

In this section, we will describe the working principle of the sky-surface wave hybrid radar and deduce the radar equation. In the equation, the most important parameter is the propagation loss. So we will discuss how to calculate it. Then, an example is given.

2.1. The Geometry of Sky-Surface Wave Hybrid Radar. The work pattern of the sky-surface wave hybrid radar is different from the sky-wave and the surface-wave OTH radar. The transmitter located far from the coast emits the electromagnetic waves and the receiver located at the coast receives the echo signal. So the radar equation should be based on the equations of sky-wave and surface-wave OTH radar equation, and the effect of the ionosphere and sea surface propagation should also be taken into consideration. The geometrical model of this radar is shown in Figure 1. For simplification, the curvature of the earth and the curve propagation in the ionosphere are not considered in this paper.

In Figure $1, L$ is the straight line distance from the transmitter to the receiver; $h$ is the vertical distance from ionospheric reflection point to the ground; $R_{1}$ is the distance from the transmitter to reflection point; $R_{2}$ is the distance from the target to reflection point; $R_{w g}$ is the straight line distance from the transmitter to the target; $R_{r}$ is the distance between receiver and the target; $\alpha$ is the emission elevation of the radar beam; $\beta$ is the bistatic angle of transmitter, receiver, and target; $\theta_{r}$ is the angle between the baseline of the transmitter to the receiver and the line of receiver to the target; $v$ is the target velocity; $\Phi$ is the angle between the target velocity direction and the bisectors of $\beta$. From the geometrical relationship, it can be found that $R_{1}=R_{2}$ and the propagation distance of the wave is $R=R_{1}+R_{2}+R_{r}$.

2.2. The Radar Equation of the Hybrid Radar. Without considering the ionospheric effect, the power density of the wave emitted by the transmitter at the target location is

$$
S_{t}=\frac{P_{t} \sigma_{t}}{4 \pi R_{s}^{2}}\left(\mathrm{~W} / \mathrm{m}^{2}\right),
$$

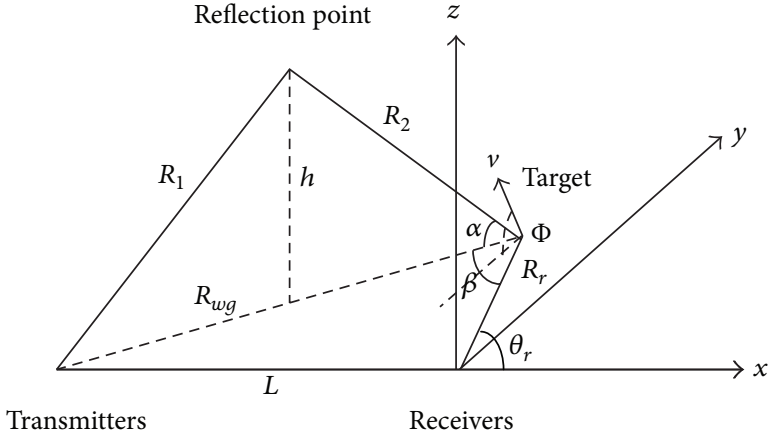

FIGURE 1: The geometrical model of the HF sky-surface hybrid radar.

where $P_{t}$ denotes the transmitting power of the sky-wave radar $(\mathrm{W})$ and $R_{s}$ denotes the propagation distance from the transmitter to the target. The scattering power at the target is

$$
P_{t 2}=S_{t} \times \sigma_{t} \times 10^{-3}=\frac{P_{t} \sigma_{t}}{4 \pi R_{s}^{2}} \times 10^{-3}(\mathrm{~kW}),
$$

where $\sigma_{t}$ is the RCS. The field intensity received by the receiver is

$$
E_{r}\left(R_{r}\right)=E\left(R_{r}\right) \sqrt{\frac{P_{t 2}}{3}}(\mu \mathrm{V} / \mathrm{m}) .
$$

The effective cross-sectional area of the receiving antenna is $A_{r}=D_{r} \lambda^{2} / 4 \pi$, where $\lambda$ is the working wavelength of the radar; then the power received by the receiving antenna is

$$
P_{r}=\frac{E_{r}^{2}\left(R_{r}\right)}{120 \pi} \times 10^{-12} \times A_{r}(\mathrm{~kW}) .
$$

$E_{r}\left(R_{r}\right)$ is the radiation field intensity $(\mu \mathrm{V} / \mathrm{m})$ where the distance to the receivers is $R_{r}$ and the transmitter is located at the coast with the transmitting power $1 \mathrm{~kW}$. Then $P_{r}$ can be written as

$$
P_{r}=\frac{5.6 \times 10^{-21} E^{2}\left(R_{r}\right) P_{t} D_{t} D_{r} \lambda^{2} \sigma_{t}}{R_{s}^{2}}(\mathrm{~W}) .
$$

A total loss factor $L$ is used to express the effects of different factors on the radar detection performance. And $L$ should include the following factors: (1) the ionospheric propagation loss $L_{p}$, which includes the absorption loss, polarization loss generated by the Faraday effect, multipath effects, and so on; (2) the radar system loss $L_{s}$, which contains the loss produced by the weighting processing, the beam forming, the antenna efficiency, and so on; (3) sea wave additional loss $L_{w}$. 


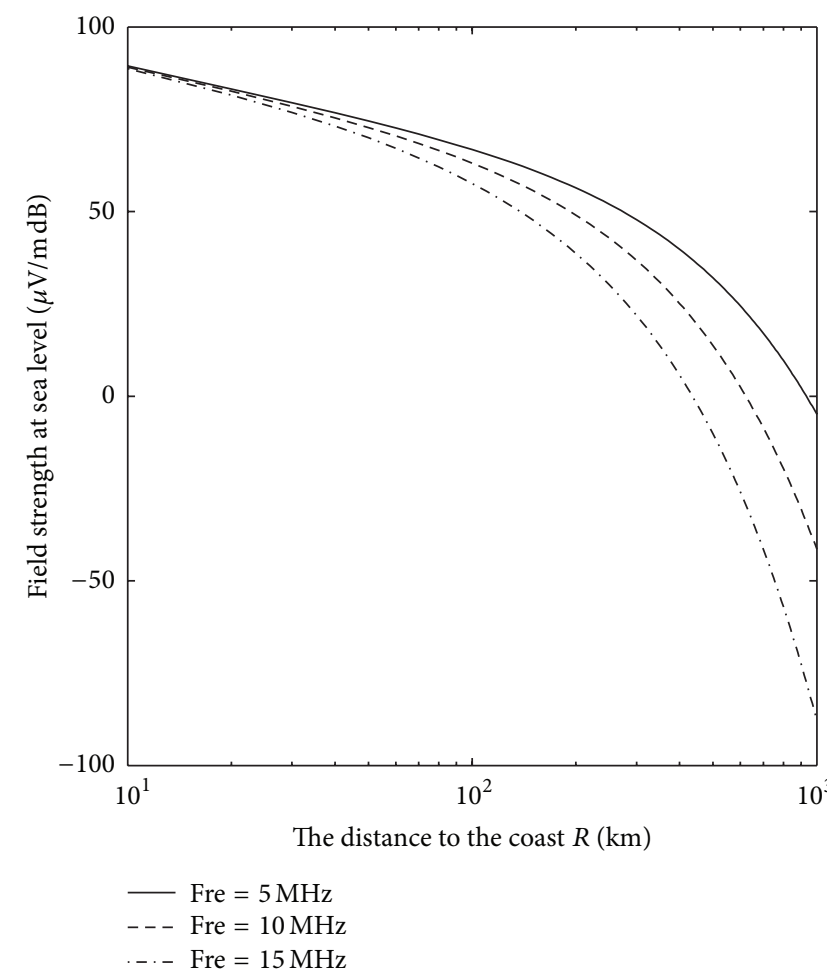

(a) The curve of $E(R)$ changes with the $R$ changes in different frequency at sea level

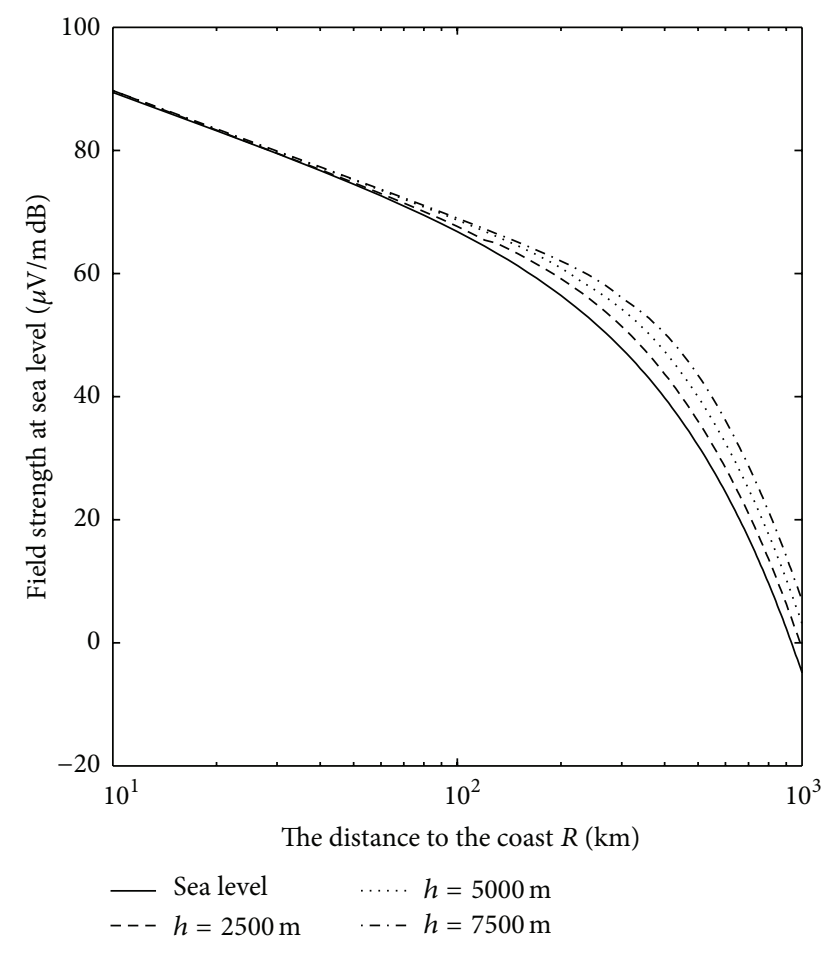

(b) The curve of $E(R)$ changes with the $R$ changes in different height when frequency is $5 \mathrm{MHz}$

FIGURE 2: The characters of $E(R)$.

If the coherent time is $T_{c}$, the received effective energy $E_{r a}$ can be written as

$$
\begin{aligned}
E_{r a}= & \frac{P_{r} \times T_{c}}{L} \\
= & 5.6 \times 10^{-21} E^{2}\left(R_{r}\right) P_{t} D_{t} D_{r} \lambda^{2} \sigma_{t} \\
& \times \frac{T_{c}}{R_{s}^{2} L_{p} L_{s} L_{w}}(\mathrm{~W}) .
\end{aligned}
$$

For the target with a low radial velocity, the signal to clutter energy ratio (SCR) should be taken into consideration in the radar equation, while for the target with a high one, the signal to noise ratio (SNR) should be considered. The power of the noise $P_{n}(f)=K T_{o} F_{a}$. Then the radar equation is

$$
\begin{aligned}
\frac{S}{N}= & \frac{E_{r a}}{P_{n}(f)} \\
= & 5.6 \times 10^{-21} E^{2}\left(R_{r}\right) P_{t} D_{t} D_{r} \lambda^{2} \sigma_{t} \\
& \times \frac{T_{c}}{R_{s}^{2} L_{p} L_{s} L_{w} K T_{o} F_{a}},
\end{aligned}
$$

where $P_{t}$ is the mean transmission power, $K$ is Boltzmann's constant, $T_{o}$ is the absolute temperature, and $F_{a}$ is the external noise factor.
To calculate (7), $E(R)$ and the system loss should be known. For $E(R)$, we can get the value in the ITU-Radio wave Propagation (Vol.2000) report, as shown in Figure 2. For the latter, the sea wave additional loss and ionospheric propagation loss are mainly considered and how to calculate them is discussed in Sections 2.3 and 2.4.

2.3. The Sea Wave Additional Loss Factor. The value of $E(R)$ in Figure 2 is gotten when the sea surface is smooth. However, the sea wave, sea wind, and the weather will cause an additional loss $L_{w}$. And $L_{w}$ is related to the radar working frequency, sea-state, and the propagation distance.

$L_{w}$ can be obtained in paper [11], as shown in Figure 3. The sea surface additional loss increases sharply as the frequency, sea wind, and propagation distance increase. To reduce the effect of $L_{w}$, the working frequency of the this hybrid radar should be selected in the low band.

Figure 3 shows $L_{w}$ varies with different sea states when the working frequency is $3 \mathrm{MHz}, 5 \mathrm{MHz}, 10 \mathrm{MHz}$, and $20 \mathrm{MHz}$. Using the interpolation method, we can approximately calculate $L_{w}$ when the frequency is $3 \sim 20 \mathrm{MHz}$ and the sea state is from state 1 to state 6 .

2.4. The Ionospheric Propagation Loss. The ionospheric propagation loss includes the polarization loss and the ionospheric 


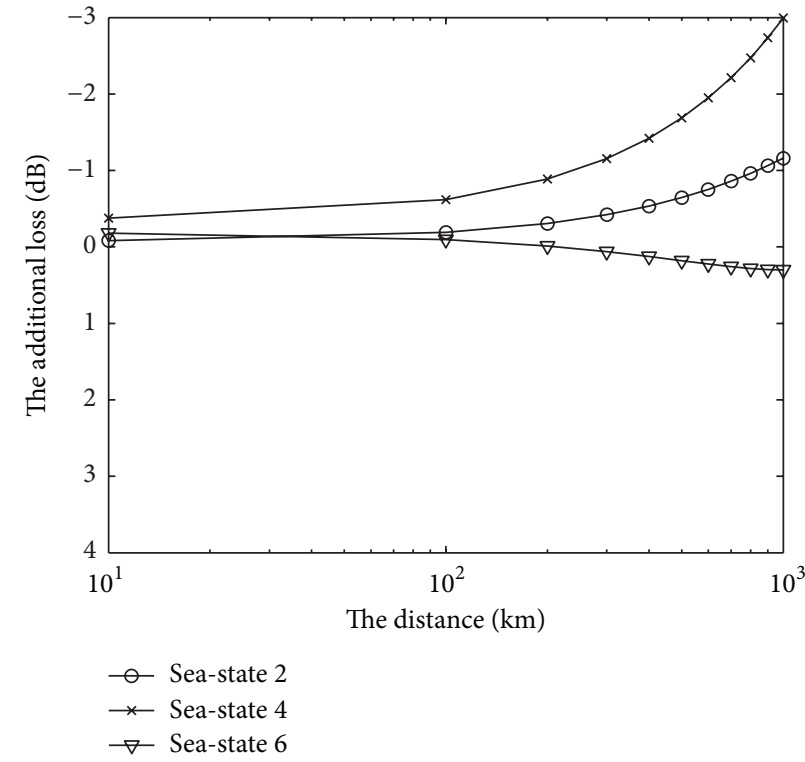

(a) $f=3 \mathrm{MHz}$

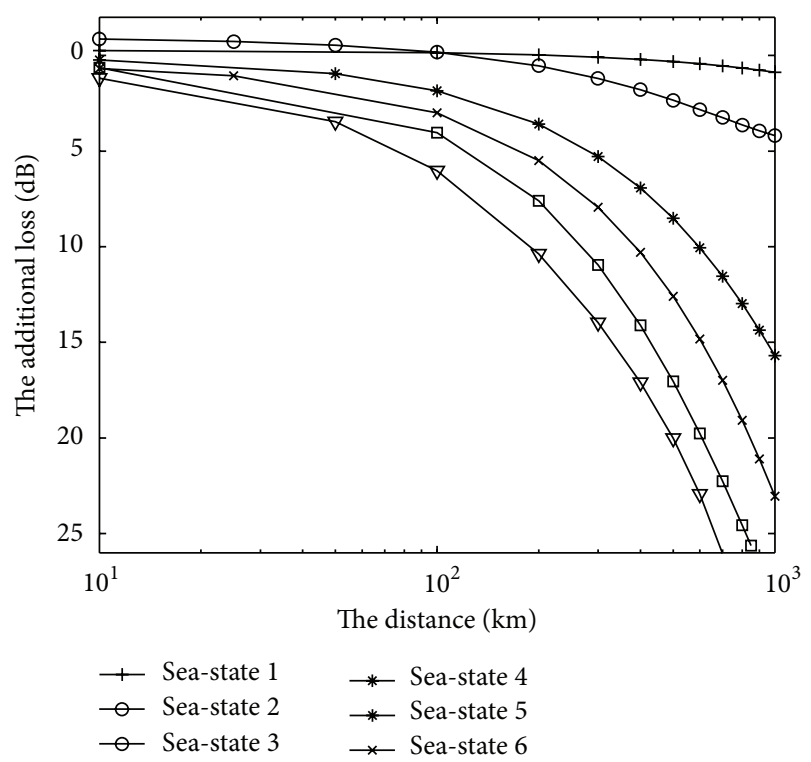

(c) $f=10 \mathrm{MHz}$

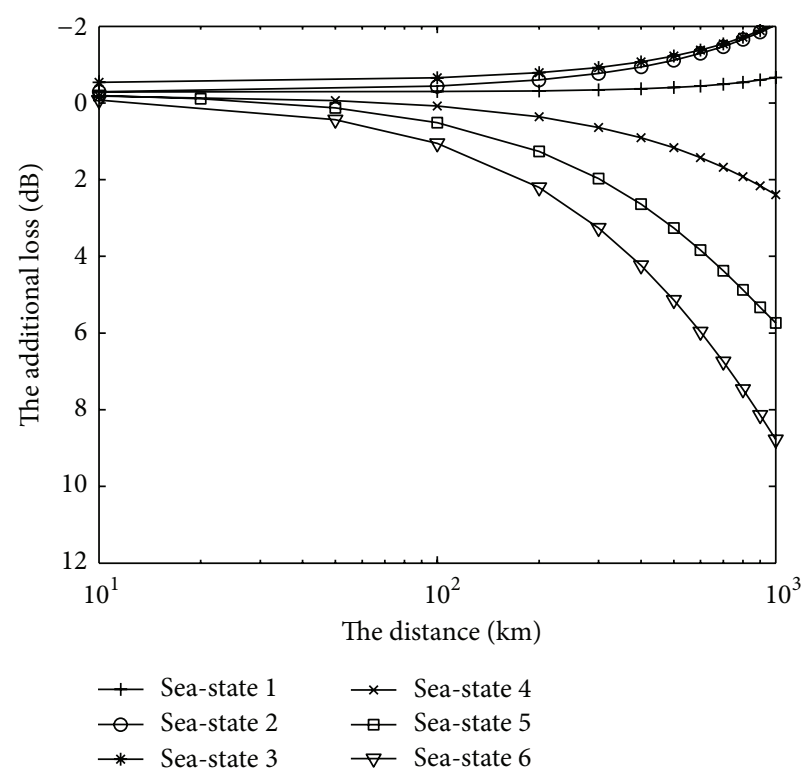

(b) $f=5 \mathrm{MHz}$

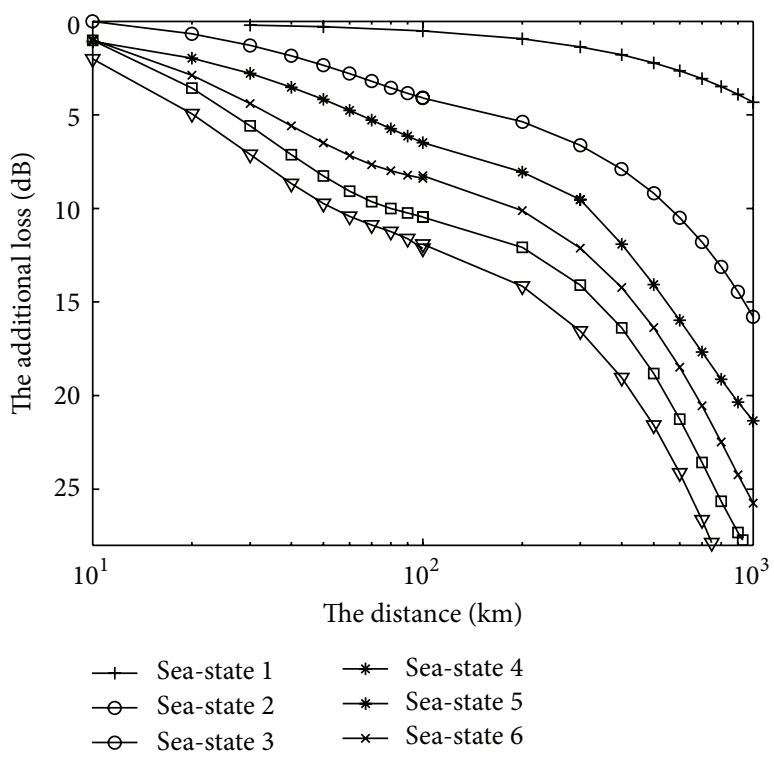

(d) $f=20 \mathrm{MHz}$

FIgURE 3: The curve of $L_{w}$ with different sea states ( $\mathrm{S}-\mathrm{T}$ is sea state; $\mathrm{W}-\mathrm{V}$ is wind velocity).

absorption loss $L$. The polarization loss is usually $3 \mathrm{~dB}$. And the ionospheric absorption loss usually is obtained with

$$
L=\frac{677.2 \sec \theta_{0}}{\left(f+f_{H}\right)^{1.98}+10.2} I .
$$

The unit of $L$ is $\mathrm{dB} . \theta_{0}$ is the incident angle at $100 \mathrm{~km}$ high, and $\theta_{0}=\arcsin (0.985 \cdot \cos \Delta)$. $\Delta$ is the launch elevation, $f$ is the working frequency $(\mathrm{MHz})$, and $f_{H}$ is the magnetic gyro frequency $(\mathrm{MHz})$ at $100 \mathrm{~km}$ high. $I$ is an absorption index [12, 13].
2.5. An Example of HF Sky-Surface Wave Hybrid Radar. Without considering the curvature of the earth, the geometrical model of this hybrid radar is shown in Figure 1. Assuming the working frequency $f_{o}=10 \mathrm{MHz}$, the straight distance between the transmitting station and the receiving station $L=$ $1000 \mathrm{~km}$ and the height from ionospheric reflection point to the ground $h=200 \mathrm{~km}$. As the detection range of the surfacewave radar is usually less than $200 \mathrm{~km}$, let $R_{r}=230 \mathrm{~km}$.

Let the transmitting gain $D_{t}=100$, let the receiving gain $D_{r}=14$, let the aircraft $\sigma_{t}=100 \mathrm{~m}^{2}$, let the coherent integration time $T_{c}=60 \mathrm{~s}$, let the radar system loss $L_{s}=10 \mathrm{~dB}$, 
$T_{o}=290 \mathrm{~K}$, let $K=1.38 \times 10^{-23}$, and let the noise factor $F_{a}=40 \mathrm{~dB}$.

When $R_{r}=230 \mathrm{~km}$ and $f_{o}=10 \mathrm{MHz}$, we can get $E(R)=$ $45.3 \mathrm{~dB} \mu \mathrm{V} / \mathrm{m}$ in Figure 2.

When the sea-state is 5 , the additional wave loss $L_{w}=$ $8.6 \mathrm{~dB}$ is calculated by the interpolation based on the data as shown in Figure 3. But the value of $L_{w}$ is the back and forth attenuation in Figure 3. As $L_{w}$ is the attenuation of the back propagation in (7), $L_{w}$ should be half of $8.6 \mathrm{~dB} . L_{w}=4.3 \mathrm{~dB}$.

By (8), the ionospheric attenuation $L=7.1 \mathrm{~dB}$. And the attenuation loss of the Faraday effect is $3 \mathrm{~dB}$. Taking these values into (7), we can get the transmitting power $P_{t}=$ $32.7 \mathrm{~kW}$, which is similar to an actual value of the OTH radar. So (8) is correct theoretically.

\section{Analysis of the Ambiguous Function of the Hybrid Radar}

We often use the ambiguity function to analyze the rangevelocity resolution of the radar system and the effect factors of the resolution. So in this section, we will discuss the ambiguous function of the hybrid radar $[14,15]$.

3.1. Calculation of the Geometrical Parameters. From Figure 1, the distance between the transmitting station to the target is

$$
\begin{aligned}
R_{w g}^{2} & =R_{r}^{2}+L^{2}-2 R_{r} L \cos \left(\pi-\theta_{r}\right) \\
& =R_{r}^{2}+L^{2}+2 R_{r} L \cos \theta_{r} .
\end{aligned}
$$

Then,

$$
\begin{aligned}
R_{1}^{2} & =h^{2}+\left(\frac{1}{2} R_{w g}\right)^{2} \\
& =h^{2}+\frac{1}{4}\left(R_{r}^{2}+L^{2}+2 R_{r} L \cos \theta_{r}\right) .
\end{aligned}
$$

The total propagation distance is

$$
R=R_{r}+2 \sqrt{h^{2}+\frac{1}{4}\left(R_{r}^{2}+L^{2}+2 R_{r} L \cos \theta_{r}\right)} .
$$

Assuming the transmitting station and the receiving station are stationary, the range rate of the target relative to the transmitting station is the velocity component along the direction of target to the ionospheric reflection point, which can be expressed as

$$
\frac{d}{d t} R_{t}=v \cos \left(\phi-\frac{\beta}{2}\right) \cos \alpha
$$

The range rate relative to the receiving station is

$$
\frac{d}{d t} R_{r}=v \cos \left(\phi+\frac{\beta}{2}\right) .
$$

Then the change rate of the total propagation distance is

$$
\begin{aligned}
\frac{d}{d t} R= & \frac{d}{d t}\left(R_{t}+R_{r}\right) \\
=v & {\left[\cos \phi \cos \left(\frac{\beta}{2}\right)(1+\cos \alpha)\right.} \\
& \left.+\sin \phi \sin \left(\frac{\beta}{2}\right)(\cos \alpha-1)\right],
\end{aligned}
$$

where

$$
\cos \beta=\frac{R_{w g}^{2}+R_{r}^{2}-L^{2}}{2 R_{w g} R_{r}}=\frac{R_{r}+L \cos \theta_{r}}{\sqrt{R_{r}^{2}+L^{2}+2 R_{r} L \cos \theta_{r}}} .
$$

The relationship between the launch elevation $\alpha$ and $\theta_{r}$ is

$$
\alpha=\arctan \left(\frac{2 h}{R_{w g}}\right)=\arctan \left(\frac{2 h}{\sqrt{R_{r}^{2}+L^{2}+2 R_{r} L \cos \theta_{r}}}\right) .
$$

Then the total propagation distance can be obtained by the initial value $R_{o}$ and the range rate. The expression is

$$
\begin{aligned}
R(t) & =R_{o}+v t=R \\
& =\left[R_{r}+2 \sqrt{D}\right] \\
& +v\left[A_{1} \sqrt{\frac{1}{2}+\frac{B}{2 \sqrt{C}}}+A_{2} \sqrt{\frac{1}{2}-\frac{B}{2 \sqrt{C}}}\right] t,
\end{aligned}
$$

where

$$
\begin{gathered}
A_{1}=\cos \phi(1+\cos \alpha), \quad A_{2}=\sin \phi(-1+\cos \alpha), \\
B=R_{r}+L \cos \theta_{r}, \quad C=R_{r}^{2}+L^{2}+2 R_{r} L \cos \theta_{r}, \\
D=h^{2}+\frac{1}{4} C .
\end{gathered}
$$

3.2. The Ambiguity Function of the Hybrid Radar. The FMCW signal is often used as the transmitting signal in the OTH radar system, which is expressed as below:

$$
S_{T}(t)=\cos \left\{2 \pi\left[f_{o} t+\frac{\mu t^{2}}{2}\right]+\phi_{o}\right\}
$$

where $T$ is the sweeping period, $\mu=B_{w} / T$ is the sweeping slope, and $B_{w}$ is the sweeping bandwidth.

For simplification, assume there are only two targets in the area that can be detected. The positions of the two targets in the radar plane are $\left(R_{r}, \theta_{r}\right)$ and $\left(R_{r}+\delta, \theta_{r}\right)$, respectively. Only the velocity component of the target along direction of the bistatic angle bisector can be detected, That is, $\Phi=0$. Let 
the velocity component along the bistatic angle of two targets be $V, V+\zeta$. Then, the echo time delay of Target 1 is

$$
\begin{aligned}
\tau_{1}(t)=\frac{R_{1}(t)}{c}= & {\left[R_{r}+2 \sqrt{D}\right] \frac{1}{c} } \\
& +\frac{V}{c} A_{1} \sqrt{\frac{1}{2}+\frac{B}{2 \sqrt{C}}} t \\
= & E_{1}+F_{1} t .
\end{aligned}
$$

The echo time delay of Target 2 is

$$
\tau_{2}(t)=\frac{R_{2}(t)}{c}=\left[R_{r}+\delta+2 \sqrt{D+\frac{1}{2} B \delta+\frac{1}{4} \delta^{2}}\right] \frac{1}{c}
$$

$$
\begin{aligned}
\xi^{2} & =\int_{-T / 2}^{T / 2}\left|S_{1}(t)-S_{2}(t)\right|^{2} d t \\
& =2 E-2 \int_{-T / 2}^{T / 2} S_{1}(t) S_{2}^{*}(t) d t \\
& =2 E-2 E \operatorname{Re}\left\{\int_{-T / 2}^{T / 2} e^{j 2 \pi\left\{\left[f_{o}\left(E_{2}-E_{1}\right)+(\mu / 2)\left(E_{1}^{2}-E_{2}^{2}\right)\right]+\left[f_{o}\left(F_{2}-F_{1}\right)+\mu\left(E_{2}-E_{1}\right)+\left(E_{1} F_{1}-E_{2} F_{2}\right)\right] t+\mu\left[\left(F_{2}-F_{1}\right)+(1 / 2)\left(F_{1}^{2}-F_{2}^{2}\right)\right] t^{2}\right\}} d t\right\},
\end{aligned}
$$

where $E=\int_{-T / 2}^{T / 2}\left|S_{1}(t)\right|^{2} d t=\int_{-T / 2}^{T / 2}\left|S_{2}(t)\right|^{2} d t=$ contant.

So $\xi$ is related to the ambiguity function $\chi(\delta, \zeta)$ :

$$
\chi(\delta, \zeta)=\int_{-T / 2}^{T / 2} e^{j 2 \pi\left\{f_{o}\left(F_{2}-F_{1}\right)+\mu\left[\left(E_{2}-E_{1}\right)+\left(E_{1} F_{1}-E_{2} F_{2}\right)\right] t+\mu\left[\left(F_{2}-F_{1}\right)+(1 / 2)\left(F_{1}^{2}-F_{2}^{2}\right)\right] t^{2}\right\}} d t \approx \int_{-T / 2}^{T / 2} e^{j 2 \pi\left\{f_{o}\left(F_{2}-F_{1}\right) t+\mu\left(E_{2}-E_{1}\right) t\right\}} d t .
$$

Equation (24) can be simplified as

$$
\begin{aligned}
& \chi(\delta, \zeta) \\
& =\sin c\left(\pi T \frac{\zeta}{\lambda}\left[A_{1} \sqrt{\frac{1}{2}+\frac{B+\delta}{2 \sqrt{C+2 B \delta+\delta^{2}}}}\right]\right. \\
& \left.\quad+\frac{\pi B_{w}}{c}\left[\delta+2 \sqrt{D+\frac{1}{2} B \delta+\frac{1}{4} \delta^{2}}-2 \sqrt{D}\right]\right) .
\end{aligned}
$$

From (25), we find that $\chi(\delta, \zeta)$ is the function of $R_{r}$ and $\theta_{r}$, which indicates that the ambiguity function is related to the location of the target.

3.3. Range Resolution. When the range resolution is considered, the velocity components of the two targets along the direction of the bistatic bisector are the same, which means $\zeta=0$. So the ambiguity function of the range resolution can be expressed as

$$
\chi(\delta, 0)=\sin c\left[\frac{\pi B_{w}}{c}\left(\delta+2 \sqrt{D+\frac{1}{2} B \delta+\frac{1}{4} \delta^{2}}-2 \sqrt{D}\right)\right] .
$$

The condition that two targets can be distinguished is $\chi(\delta, 0) \geq-3 \mathrm{~dB}$, so

$$
\frac{B_{w}}{c}\left(|\delta|+2 \sqrt{D+\frac{1}{2} B|\delta|+\frac{1}{4} \delta^{2}}-2 \sqrt{D}\right) \leq a,
$$

where $a=0.443$. Then (27) can be written as

$$
\begin{aligned}
2 \delta^{2}+ & 2 B|\delta|+4 \sqrt{D+\frac{1}{2} B|\delta|+\frac{1}{4} \delta^{2}} \\
& \leq \frac{4 a c \sqrt{D}}{B_{w}}+\frac{a^{2} c^{2}}{B_{w}^{2}} .
\end{aligned}
$$

As $2 \delta^{2} \ll 2 B|\delta|+4 \sqrt{D+(1 / 2) B|\delta|+(1 / 4) \delta^{2}}(1 / 2),(1 / 2) B|\delta|$ $+(1 / 4) \delta^{2} \ll D, a^{2} c^{2} / B_{w}^{2} \ll 4 a c \sqrt{D} / B_{w}$, and $2|\delta| \leq \delta_{R}$, so the radar range resolution $\delta_{R}$ is

$$
\delta_{R}=\frac{4 a c}{B_{w}(B / \sqrt{D}+2)}
$$

$$
=4 a c
$$




$$
\begin{aligned}
\times\left(B_{w}(\right. & \left(R_{r}+L \cos \theta_{r}\right) \\
& \left.\left.\div \sqrt{h^{2}+\frac{1}{4}\left(R_{r}^{2}+L^{2}+2 R_{r} L \cos \theta_{r}\right)}+2\right)\right)^{-1}
\end{aligned}
$$

From (29), it can be known that the range resolution of the hybrid radar is not only inversely proportional to the transmitting bandwidth, but also related to the location of the target and the height of reflection points in the ionosphere. Figure 4 shows the simulation results of $\delta_{R}$ with the working frequency is $15 \mathrm{MHz}$ and the bandwidth is $20 \mathrm{kHz}$.

As shown in Figure 4(b), the range resolution increases when $\theta_{r}$ increases. And when $\theta_{r} \geq 30^{\circ}$, the increasing rate increases significantly. Figures $4(\mathrm{c})$ and $4(\mathrm{~d})$ illustrate that the range resolution increases with $R_{r}$ decreases and $h$ increases respectively. We also find that the changes of $h$ and $R_{r}$ have little effect on the range resolution when $\theta_{r}$ is small. Only when $\theta_{r}$ is large, the changes have an obvious effect.
The range resolution of sky-wave or surface-wave radar is $\Delta R=c / 2 B$, which means the range resolution is only related to the bandwidth. When $B=20 \mathrm{kHz}, \Delta R=$ $7.5 \mathrm{~km}$. So, from Figure 4(b), when $\theta_{r}<44^{\circ}$, the hybrid radar has a better range resolution than monostatic OHT radar.

3.4. The Velocity Resolution. When the velocity resolution is considered, the location of the two targets are same, which means $\delta=0$. So the ambiguity function of the velocity resolution can be expressed as

$$
\chi(0, \zeta)=\sin c\left[\pi T \frac{\zeta}{\lambda}\left(A_{1} \sqrt{\frac{1}{2}+\frac{B}{2 \sqrt{C}}}\right)\right] .
$$

As the condition that two targets can be distinguished is $\chi(0, \zeta) \geq-3 \mathrm{~dB}$, so

$$
T \frac{|\zeta|}{\lambda}\left[A_{1} \sqrt{\frac{1}{2}+\frac{B}{2 \sqrt{C}}}\right] \leq a .
$$

As $2|\zeta| \leq \zeta_{v}$, the velocity resolution $\zeta_{v}$ is

$$
\begin{aligned}
\zeta_{v} & \approx \frac{2 a \lambda}{T} \frac{1}{A_{1} \sqrt{1 / 2+B / 2 \sqrt{C}}} \\
& =\frac{2 a \lambda}{T}\left(1 \times\left(1+\arctan \left(\frac{2 h}{\sqrt{R_{r}^{2}+L^{2}+2 R_{r} L \cos \theta_{r}}}\right) \sqrt{\frac{1}{2}+\frac{R_{r}+L \cos \theta_{r}}{2 \sqrt{R_{r}^{2}+L^{2}+2 R_{r} L \cos \theta_{r}}}}\right)^{-1}\right) .
\end{aligned}
$$

By (32), it can be known that the velocity resolution of the hybrid radar is not only inversely proportional to the sweep period and proportional to the wavelength, but also related to the location of the target and the height of reflection points in the ionosphere. Figure 5 shows the simulation results about $\zeta_{v}$ with $T=1 \mathrm{~s}$ and $f=15$ $\mathrm{MHz}$.

As shown in Figure 5, we can get the similar conclusion of the velocity resolution with the one about the range resolution.

For the velocity resolution of the sky-wave or surfacewave radar is $\Delta V=\lambda / 2 T, \Delta V$ is only related to the sweeping period. When $T=1 \mathrm{~s}, \Delta V=10 \mathrm{~m} / \mathrm{s}$. In comparison with Figure 5(b), when $\theta_{r}<60.7^{\circ}$, the hybrid radar has a better velocity resolution than the monostatic OHT radar.

3.5. The Coupling Analysis of Range and Velocity. From the ambiguity theory [16], when the velocity and range of the two targets are not same, the time difference and frequency difference should be different. But under some conditions, $\chi(\delta, \zeta)=0$ may occur, which means that two targets cannot be distinguished. By (25), let $\chi(\delta, \zeta)=0$; it can be obtained that

$$
\begin{aligned}
T \zeta & \left.A_{1} \sqrt{\frac{1}{2}+\frac{B+\delta}{2 \sqrt{C+2 B \delta+\delta^{2}}}}\right] \\
& +\frac{B_{w}}{c}\left[\delta+2 \sqrt{D+\frac{1}{2} B \delta+\frac{1}{4} \delta^{2}}-2 \sqrt{D}\right]=0 .
\end{aligned}
$$

The equation above is rewritten as

$$
\begin{aligned}
(2 B+ & \left.4 \sqrt{D+\frac{1}{2} B \delta+\frac{1}{4} \delta^{2}}\right) \delta+\frac{5}{4} \delta^{2} \\
= & -\frac{4 T c \zeta}{\lambda B_{w}} A_{1} \sqrt{\frac{1}{2}+\frac{B+\delta}{2 \sqrt{C+2 B \delta+\delta^{2}}}} \\
& +\left(\frac{T c \zeta}{\lambda B_{w}} A_{1}\right)^{2}\left(\frac{1}{2}+\frac{B+\delta}{2 \sqrt{C+2 B \delta+\delta^{2}}}\right) .
\end{aligned}
$$



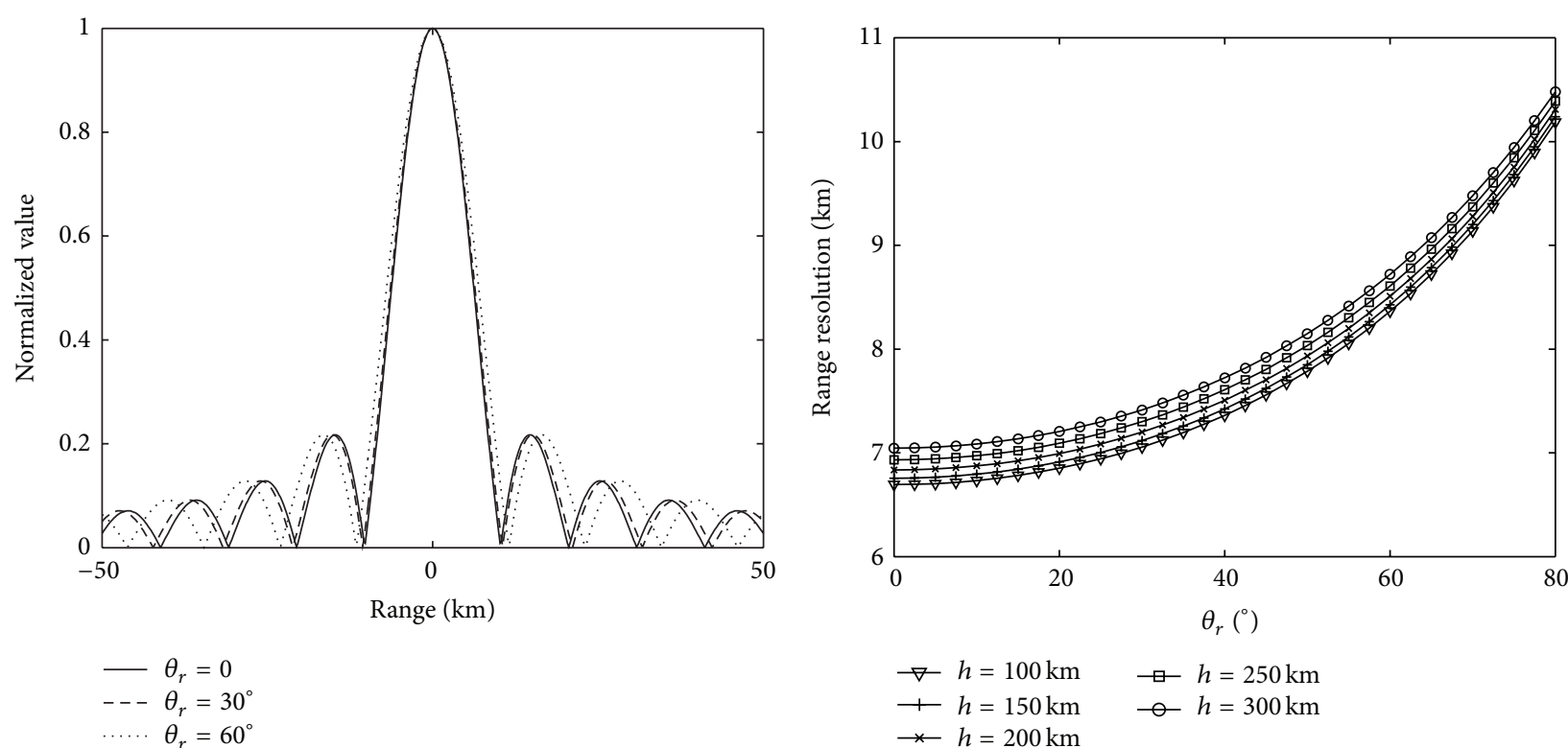

(a) The range ambiguous function when $\theta_{r}$ is $0,30^{\circ}$, or $60^{\circ}$

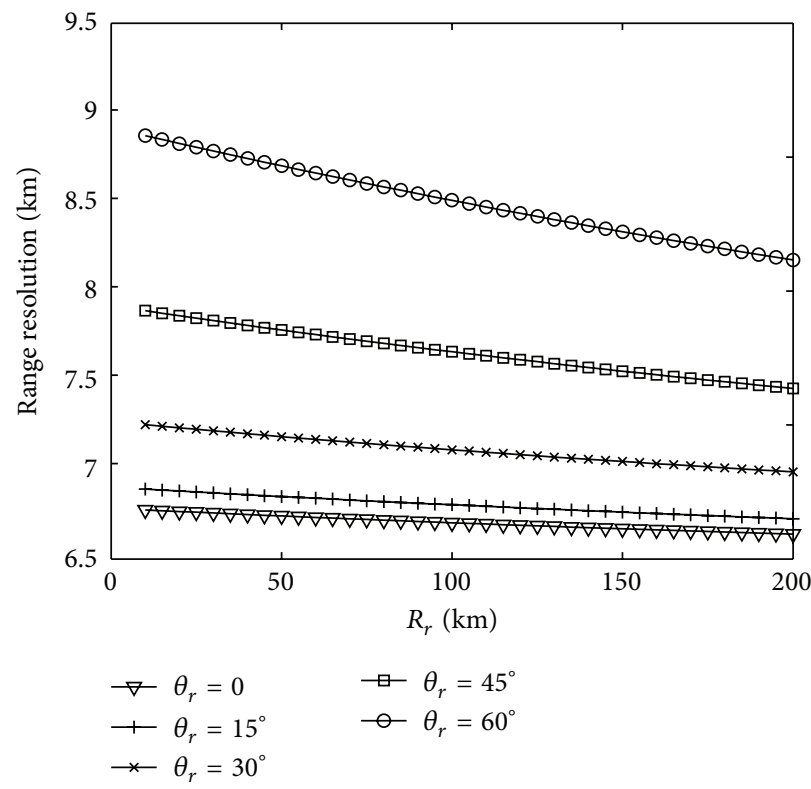

(c) When $h=200 \mathrm{~km}$, the range resolution changes with $h$ and $\theta_{r}$

(b) When $R_{r}=150 \mathrm{~km}$, the range resolution changes with $\theta_{r}$ and $h$

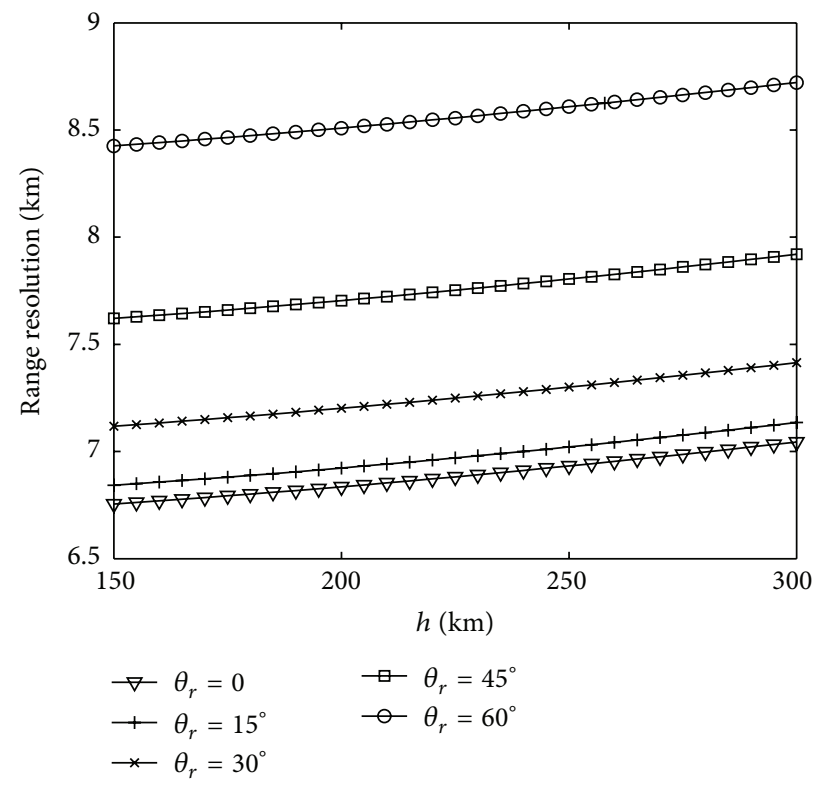

(d) When $R_{r}=150 \mathrm{~km}$, the range resolution changes with $h$ and $\theta_{r}$

FIgURE 4: The ambiguous function and the range resolution of the hybrid radar.

The second term of the left side of (34) can be ignored compared with the first term, and $\sqrt{D+(1 / 2) B \delta+(1 / 4) \delta^{2}} \approx$ $\sqrt{D}, \sqrt{1 / 2+(B+\delta) / 2 \sqrt{C+2 B \delta+\delta^{2}}} \approx \cos (\beta / 2)$, so

$$
\frac{\delta}{\zeta} \approx-\frac{4 T c \zeta}{\lambda B_{w}} \frac{1}{2 B / \sqrt{D}} \cos \left(\frac{\beta}{2}\right) .
$$

When the difference of the range-velocity between the two targets satisfies (35), the two targets cannot be distinguished.

\section{Conclusion}

As a new type of bistatic radar, the hybrid sky-surface wave OTH radar is different with the sky-wave and the surface-wave OTH radar. So the radar equation and the range-velocity resolution, the two aspects of the detection performance of this hybrid radar, are studied in this paper. According to the ones of the sky-wave and the surface-wave radar, the radar equation of this hybrid radar which concludes the ionospheric loss and the wave additional loss is derived. Then, an example is given to testify the validity. From the 


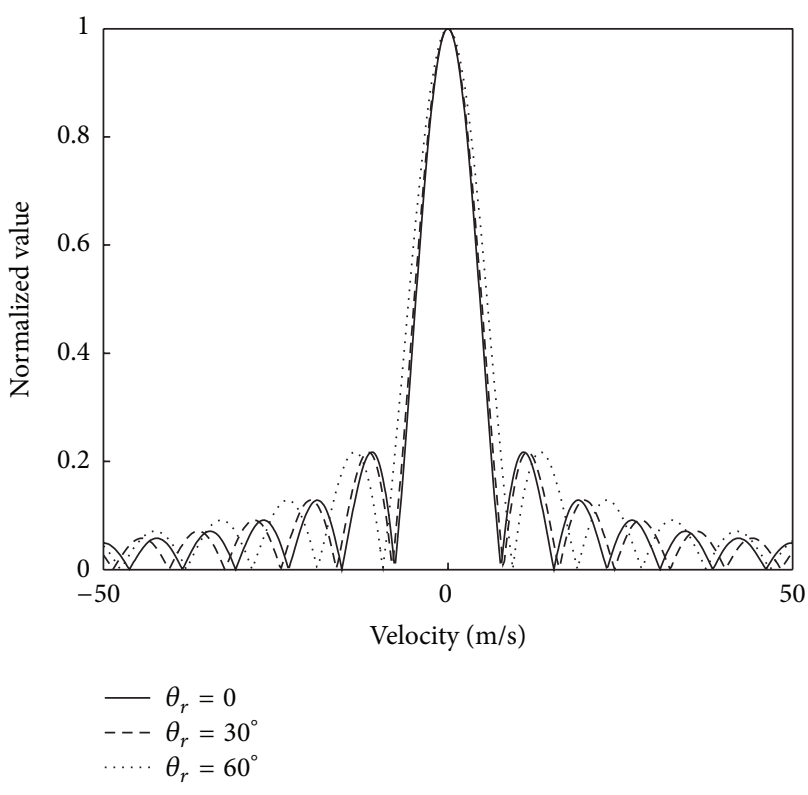

(a) The velocity ambiguous function when $\theta_{r}$ is $0,30^{\circ}$, or $60^{\circ}$

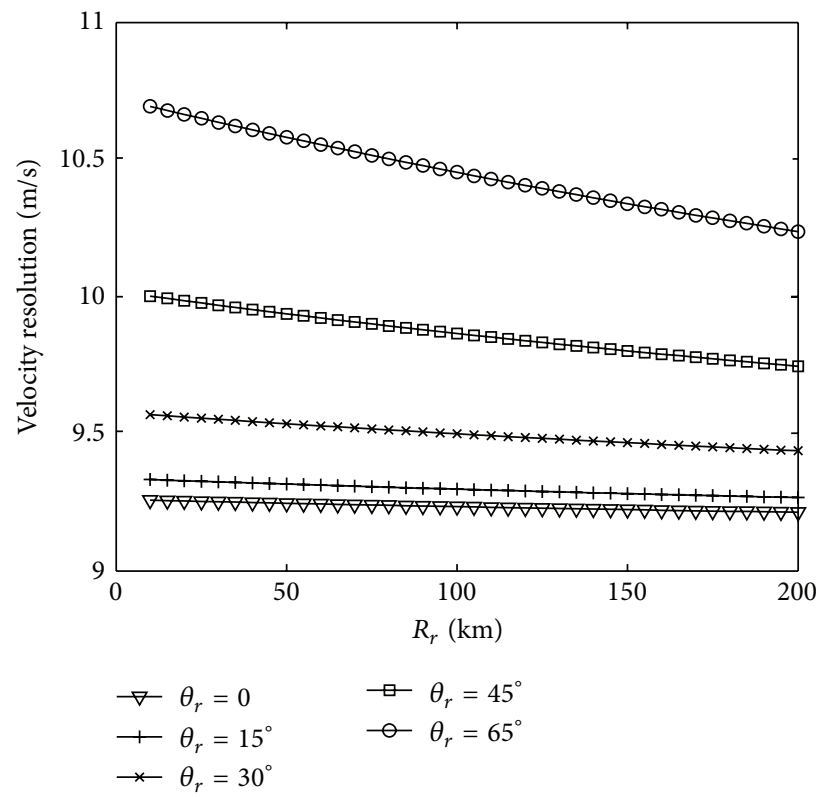

(c) When $h=200 \mathrm{~km}$, the velocity resolution changes with $h$ and $\theta_{r}$

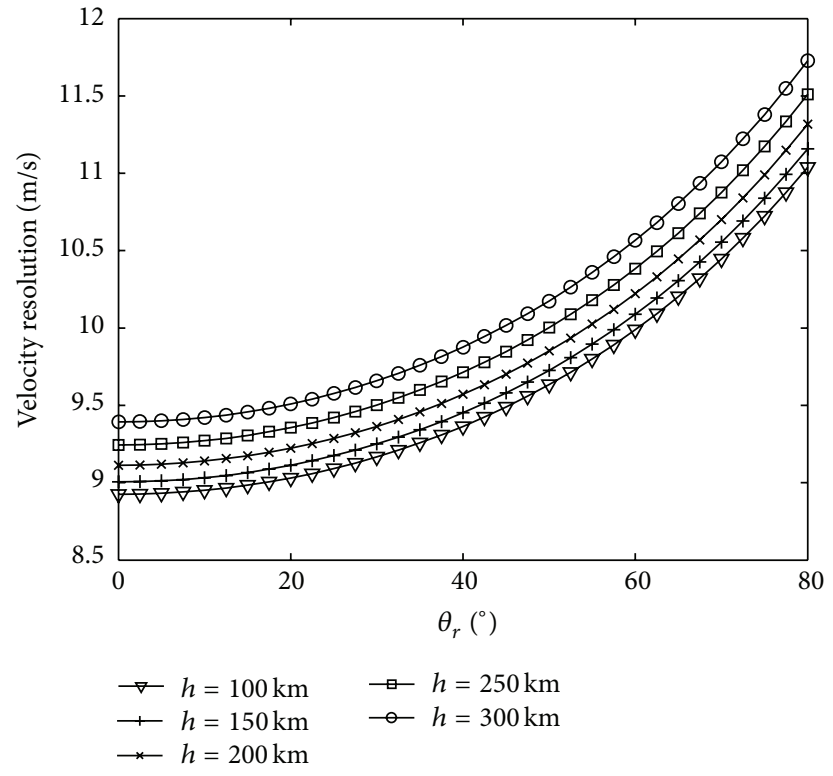

(b) When $R_{r}=150 \mathrm{~km}$, the velocity resolution changes with $\theta_{r}$ and $h$

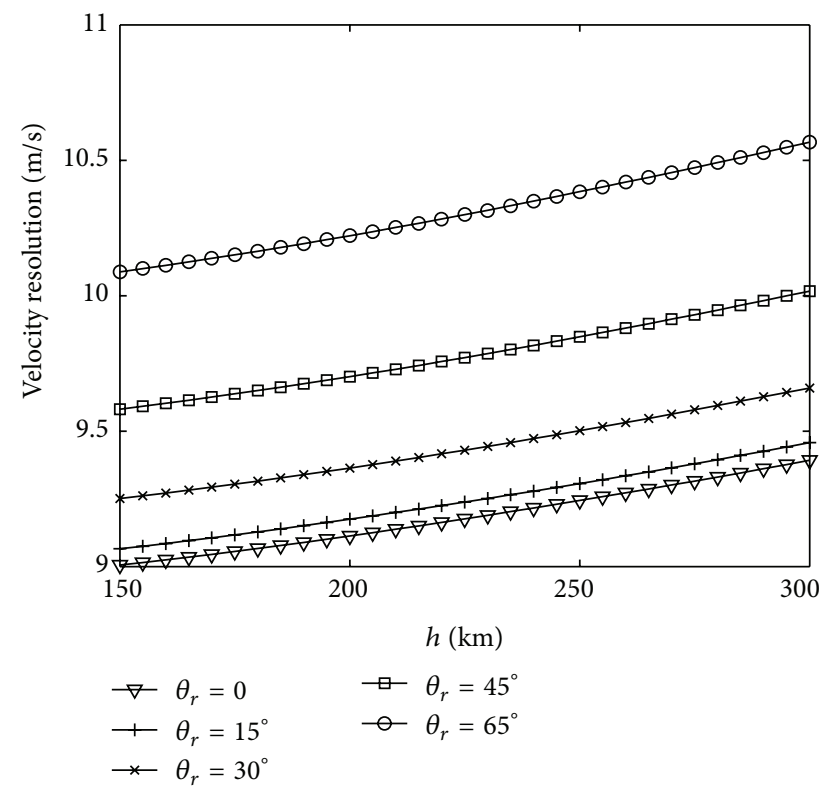

(d) When $R_{r}=150 \mathrm{~km}$, the velocity resolution changes with $h$ and $\theta_{r}$

FIgURE 5: The ambiguous function and the velocity resolution of the hybrid radar.

analysis of the ambiguity function, we find that the rangevelocity resolution of this radar is related to the location of the target and the reflection height in the ionosphere. In a certain launch elevation, the range-velocity resolution is better with respect to the one of the monostatic OHT radar, and the effect of the target location and the ionospheric reflection height can be ignored to some extent. It can be predicted that with more MIMO radar theory is applied to this radar system, the performance of the OHT radar will be further enhanced.

In this paper, the detectability of the radar system with one sky-wave radar transmitter and one surface-wave radar receiver is studied. How to evaluate the performance of the netted radar system with multiple surface-wave radar receivers and networked control [17] will be the future work.

\section{Conflict of Interests}

The authors declare that there is no conflict of interests regarding the publication of this paper.

\section{Acknowledgment}

At the point of finishing this paper, the author would like to express his sincere thanks to the National Natural Science 
Foundation (no. 61201303) and the Fundamental Research Funds for the Central Universities (HIT.NSRIF.2013027).

\section{References}

[1] E. Fishler, A. Haimovich, R. Blum, D. Chizhik, L. Cimini, and R. Valenzuela, "MIMO radar: an idea whose time has come," in Proceedings of the IEEE Radar Conference, pp. 71-78, IEEE Press, Philadelphia, Pa, USA, April 2004.

[2] R. J. Riddolls, "Limits on the detection of low-Doppler targets by a high frequency hybrid sky-surface wave radar system," in Proceedings of the IEEE Radar Conference (RADAR '08), pp. 1-4, Rome, Italy, May 2008.

[3] P. Vouras and B. Freburger, "Application of adaptive beamforming techniques to HF radar," in Proceedings of the IEEE Radar Conference (RADAR '08), pp. 795-800, May 2008.

[4] G. J. Frazer, "Forward-based receiver augmentation for OTHR," in Proceedings of the IEEE Radar Conference, pp. 373-378, Boston, Mass, USA, April 2007.

[5] Y. Li and Y. Wei, "Analysis of first-order sea clutter spectrum characteristics for HF sky-surface wave radar," in Proceedings of the International Conference on Radar (Radar '13), pp. 368-373, Adelaide, Australia, September 2013.

[6] Y. Li, Y. Wei, R. Xu, and Z. Wang, "Influence of ionosphere on resolution cell of HF hybrid sky-surface wave radar," in Proceedings of the IEEE Antennas and Propagation Society International Symposium (APSURSI '13), pp. 1028-1029, 2013.

[7] L. Zhang and H. Gao, "Asynchronously switched control of switched linear systems with average dwell time," Automatica, vol. 46, no. 5, pp. 953-958, 2010.

[8] Z. Zhixin, W. Xianrong, Z. Delei, and C. Feng, "An experimental study of HF passive bistatic radar via hybrid sky-surface wave mode," Transactions on Antennas and Propagation, vol. 61, no. 1, pp. 415-424, 2013.

[9] Y. Longquan, F. Junmei, J. Peinan, and C. Hongyan, "Time availability of hybrid sky-surface wave radar," Chinese Journal of Radio Science, vol. 28, no. 5, pp. 829-834, 840, 2013.

[10] L. Zhang, N. Cui, M. Liu, and Y. Zhao, "Asynchronous filtering of discrete-time switched linear systems with average dwell time," IEEE Transactions on Circuits and Systems I: Regular Papers, vol. 58, no. 5, pp. 1109-1118, 2011.

[11] Z. Wenyu and J. Peinan, OTH Radar Technology, Publishing House of Electronics Industry, 2004.

[12] L. Jiaying and P. Jiao, "Nomograms for tactical skywave communication in tropical forest regions," Chinese Journal of Radar Science, vol. 9, no. 1, pp. 63-72, 1994.

[13] V. Jodalen and E. V. Thrane, "A study of the relation between ionospheric absorption and predicted HF propagation parameters at high latitudes," in Proceedings of the 6th International Conference on HF Radio Systems and Techniques, pp. 64-68, New York, NY, USA, July 1994.

[14] M. C. Jackson, “ The geometry of bistatic systems," IEE Proceedings, vol. 133, no. 7, pp. 604-612, 1986.

[15] D. Trizna and J. Gordon, "Results of a bistatic HF radar surface wave sea scatter experiment," in Proceedings of the IEEE International Geoscience and Remote Sensing Symposium (IGARSS '02), vol. 3, pp. 1902-1904, Piscataway, NJ, USA, June 2002.

[16] T. Tsao, M. Slamani, P. Varshney, D. Weiner, H. Schwarzlander, and S. Borek, "Ambiguity function for a bistatic radar," IEEE
Transactions on Aerospace and Electronic Systems, vol. 33, no. 3, pp. 1041-1051, 1997.

[17] L. Zhang, H. Gao, and O. Kaynak, "Network-induced constraints in networked control systems-a survey," IEEE Transactions on Industrial Informatics, vol. 9, no. 1, pp. 403-416, 2013. 


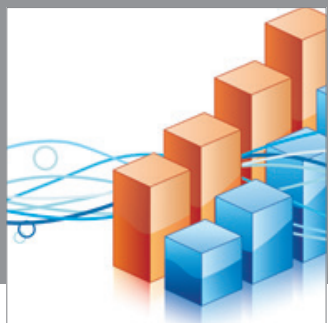

Advances in

Operations Research

mansans

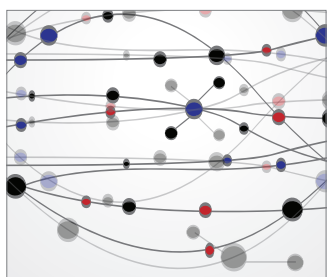

The Scientific World Journal
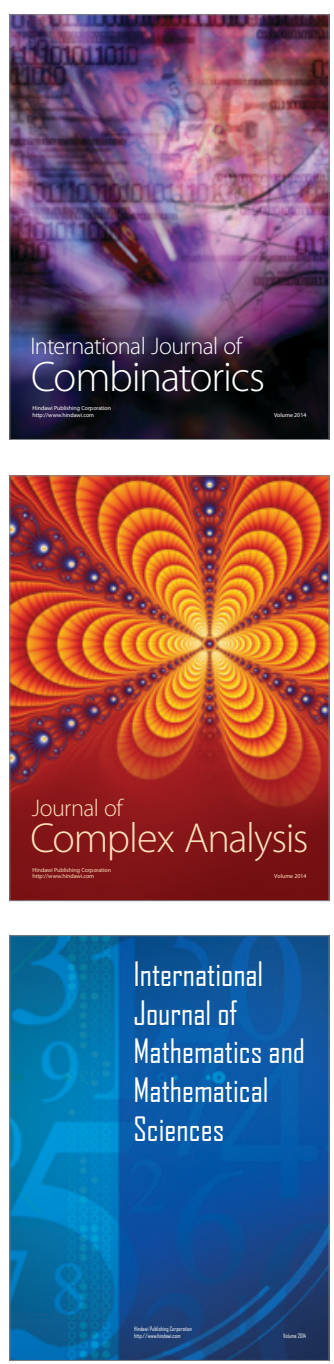
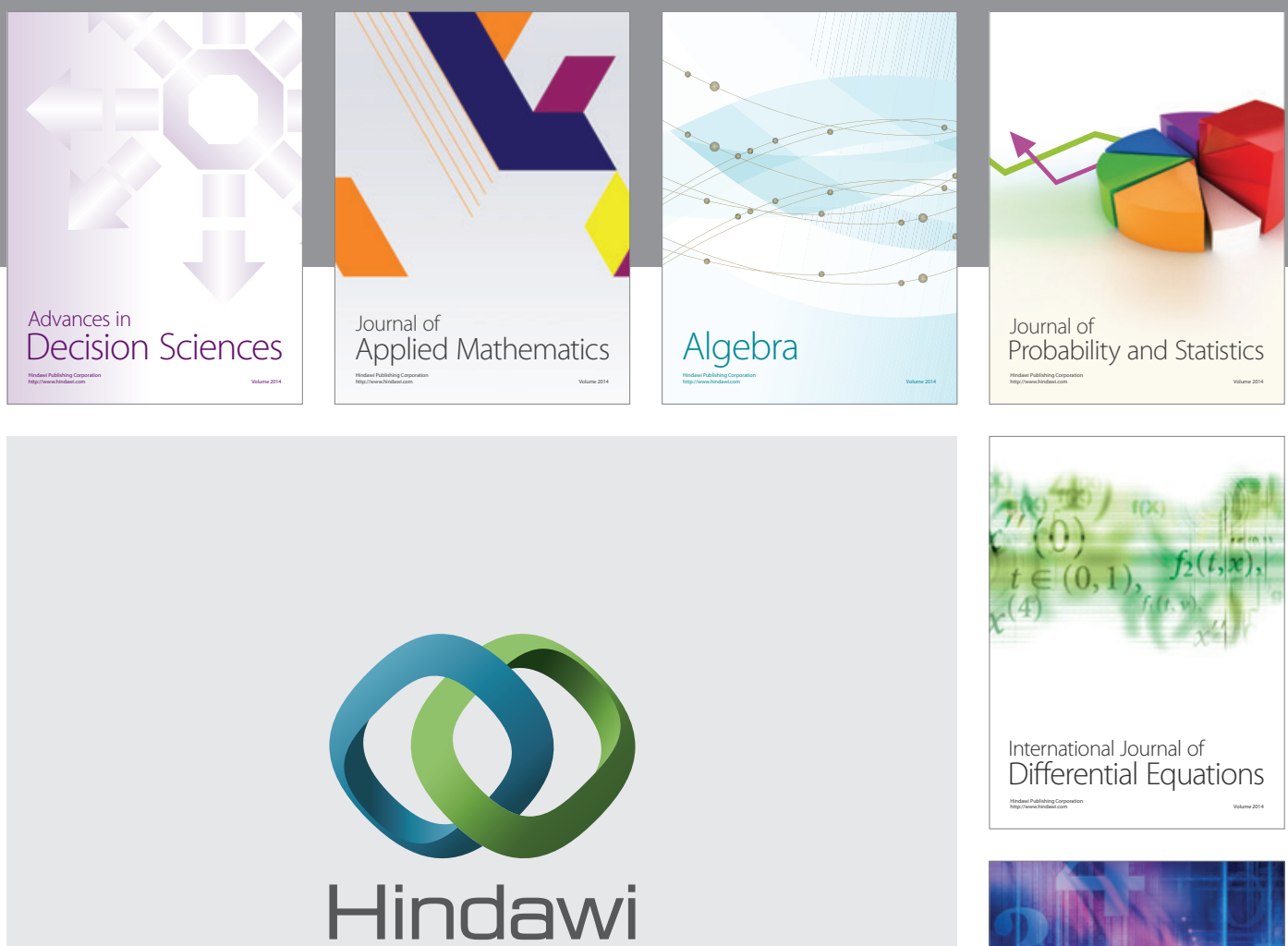

Submit your manuscripts at http://www.hindawi.com
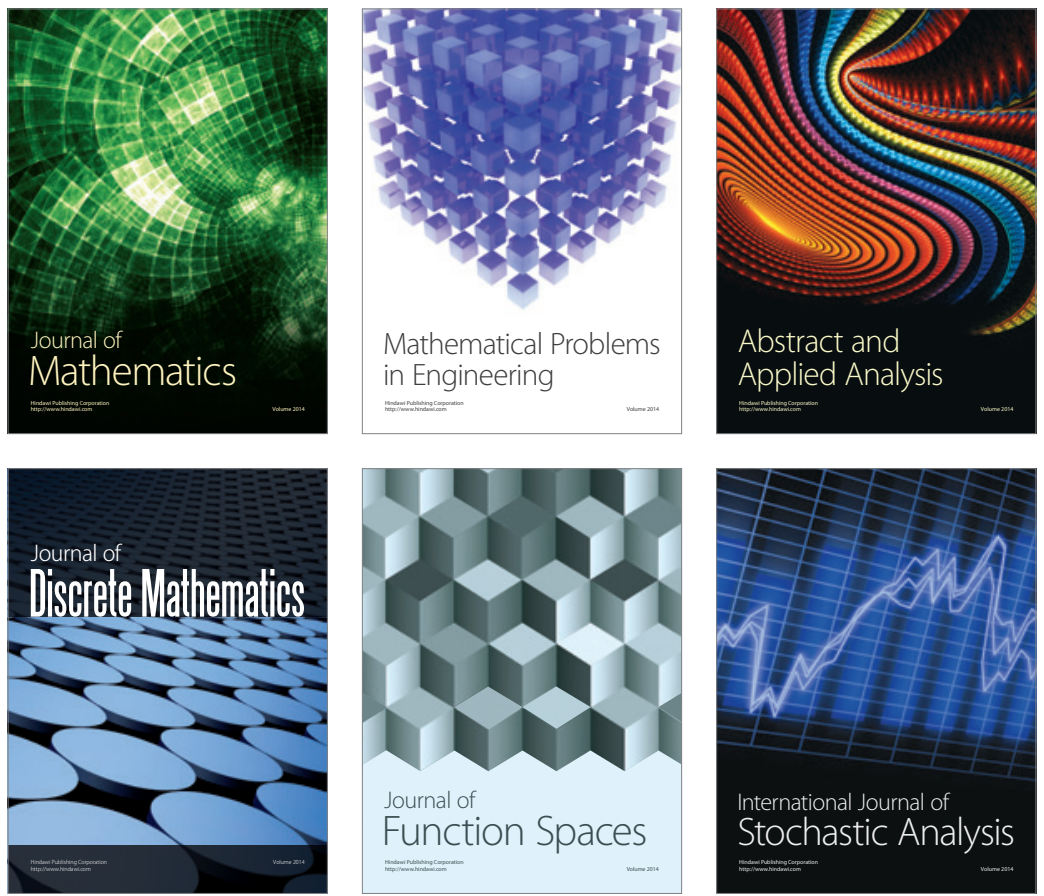

Journal of

Function Spaces

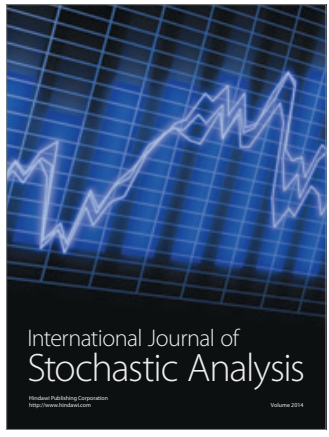

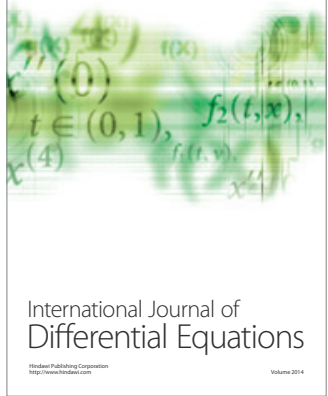
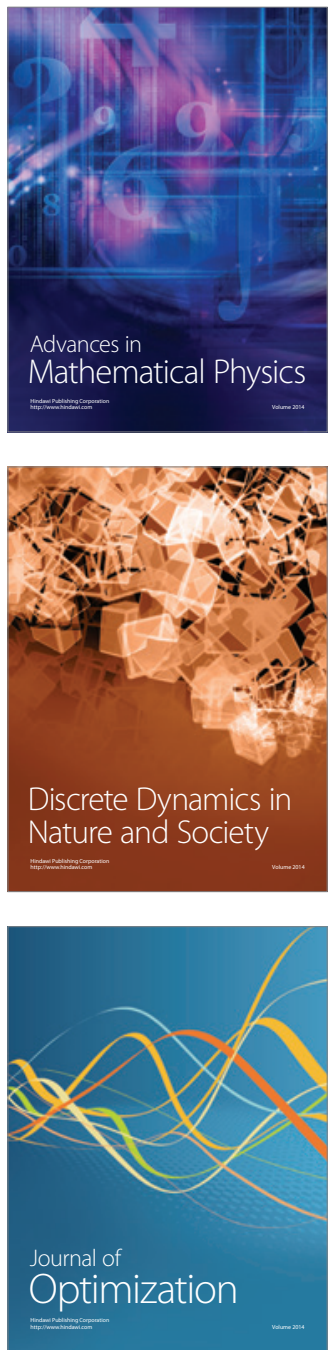